\title{
Nanocrystalline hexagonal shaped CdS thin films for photoconducting application
}

\author{
S. M. Patil, P. H. Pawar* \\ Z. B. Patil Arts, Science and Commerce College, Dhule - 424 002, India \\ *E-mail address: sham_nilima@rediffmail.com
}

\begin{abstract}
Nanocrystalline thin films of cadmium sulphide were prepared by chemical bath deposition technique onto glass substrate at $60{ }^{\circ} \mathrm{C}$. The deposition parameters were optimized to obtain good quality of nanocrystalline thin films such as, time, precursor concentration, temperature of deposition and $\mathrm{pH}$ of the solution. The studies on crystal structure, composition, surface morphology, electrical conductivity and photoconductivity of the films were carried out by using different analytical technique. Characterization includes X-ray diffraction (XRD), Field emission scanning electron microscopy (FE-SEM), Energy dispersive X-ray analysis (EDAX), Electrical and photoconductivity. The response and recovery time of the thin film were measured and presented.
\end{abstract}

Keywords: Nanocrystalline CdS; thin films; chemical bath deposition; analytical technique; photoconductivity

\section{INTRODUCTION}

Now a day's semiconducting thin film have great application due to their electrical and optical properties in the fields such as solar selective coatings, infrared detectors, solar control optoconductors, solid state, photoelectrical solar cells [1]. Cadmium sulphide is a wide band gap semiconductor $(\mathrm{Eg}=2.4 \mathrm{eV})$ and in a form of thin film is mostly obtained by vacuum methods. Thin films of II-VI semiconductors (e.g. CdS, ZnS, CdSe, ZnSe) are important for their applications in solid-state solar cells, optical coatings, optoelectronic devices and light emitting diodes [2].

Various techniques have been reported to deposit CdS thin films that include silar [3], chemical bath deposition [4], co-precipitation technique [5]. Among these chemical bath deposition technique (CBD) is a simple which is also used to deposited the semiconductor on photovoltaic device [6].

Chemical bath deposition is the simplest, capable of large area fabrication at a low cost. This process has the added advantages that material wastage is minimum and there is no need to handle poisonous gases. Though this technique has been used for quite some time for the preparation of many binary semiconductors like $\mathrm{CdS}, \mathrm{CdSe}, \mathrm{CdTe}, \mathrm{PbS}, \mathrm{ZnSe}, \mathrm{ZnS}$, its potential has not been fully utilized in developing phase, ternary, quaternary compound semiconductors probably because of the difficulty in controlling the process to get good 
quality films in a reproducible way. In the present investigation, we used CBD process to deposit solar-grade thin films on glass substrate.

Efforts are made in the present article to synthesize CdS on glass substrate using CBD, the deposition time is varied from $60-80 \mathrm{~min}$. which result the film deposition of various thickness. The parameters are optimized for uniform deposition of the films. The effect of thickness on structural, microstructure and electrical properties of this material was studied. Various characterization techniques such as XRD, FE-SEM were employed to study the thin films.

\section{EXPERIMENTAL}

\section{1. Cleaning of substrates}

The deposition was carried out using glass substrate of the dimension $75 \mathrm{~mm} \times 25 \mathrm{~mm}$ $\times 2 \mathrm{~mm}$. These substrate were first washed with distilled water and then boiled in chromic acid and again boiled in distilled water, finally the substrates were washed with distilled water [7].

\section{2. Preparation of nanocrystalline CdS thin films}

The chemical bath deposition technique was used to deposit the thin films of cadmium sulphide on glass substrate. The starting materials used were cadmium sulphate and thiourea (Made: Sd-fine $99.99 \%$ purified). Triethanolamine (TEA) was used as a complexing agent. Ammonia solutions were used to adjust $\mathrm{pH}$ of the reaction mixture. In order to obtain good quality of thin films, following parameter were adjust such as time, temperature of deposition and $\mathrm{pH}$ of the solution. The optimum value of time, temperature and $\mathrm{pH}$ were tabulated in Table 1.

Table 1. Optimum parameter to obtain nanocrystalline CdS thin films.

\begin{tabular}{|c|c|}
\hline Depositon parameter & Optimum value / item \\
\hline Deposition time & 60 min, 70 min and $80 \mathrm{~min}$ \\
$\mathrm{pH}$ & 10 \\
Cadmium sulphate, Thiourea & $0.1 \mathrm{M}$ \\
\hline Solvent & Deionized water \\
\hline Deposition temperature & $60{ }^{\circ} \mathrm{C}$ \\
\hline
\end{tabular}




\section{3. Determination of film thickness}

The film thickness was measured by a weight difference method [8] in which weight of the sample, area and densities were considered. The thickness, sample weight and sample area are related as:

$$
t=M / A \cdot \rho
$$

where,

$M$ is the weight of the sample in $g m$,

A the area of the sample in $\mathrm{cm}^{2}$

and $\rho$ the materials density in $\mathrm{gm}^{-3}$.

The thickness of the film was 765-810 nm. The values of the film thickness with $\mathrm{pH}$ of thin films are given in Table 3.

\section{4. Characterization of thin films}

The structural properties of thin films were investigated by X-ray diffraction (XRD) using $\mathrm{CuK} \alpha(\lambda=1.542 \AA)$ radiation. The Field emission scanning electron microscopic studies were carried out using JEOL model, JSM-6360A FE-SEM. Elemental analysis was carried out with an EDAX using Energy Dispersive X-ray spectrophotometer (EDS). Electrical conductivity of the films was measured by using DC two-probe method in the temperature range $313-423 \mathrm{~K}$.

\section{RESULTS AND DISCUSSION}

\section{1. Structural analysis}

Figure 1, shows the X-ray diffractogram of the CdS thin films scanned in the $2 \theta$ range of $20-80^{\circ}$. The peaks (100), (002), (101), (102), (110) and (103) corresponds to CdS (JCPDS file no. 41-1049). Major peaks are matching with standard JCPDS data of CdS. The (002) peak predominates indicating a preferential growth. This means that the grains have $c$-axis perpendicular to the substrate surface. The average crystallite size of nanostuctured CdS thin film samples were calculated by using the Scherrer formula,

$$
D=0 \cdot 9 \lambda / \beta \cos \theta
$$

where;

$D=$ Average crystallite size

$\lambda=\mathrm{X}$-ray wavelength $(1 \cdot 542 \AA)$

$\beta=$ FWHM of the peak

$\theta=$ Diffraction peak position.

The average crystallite size was presented in Table 2. Dislocation density values were calculated using the standard relations :

$$
\text { Dislocation density }(\delta)=1 / D^{2}
$$




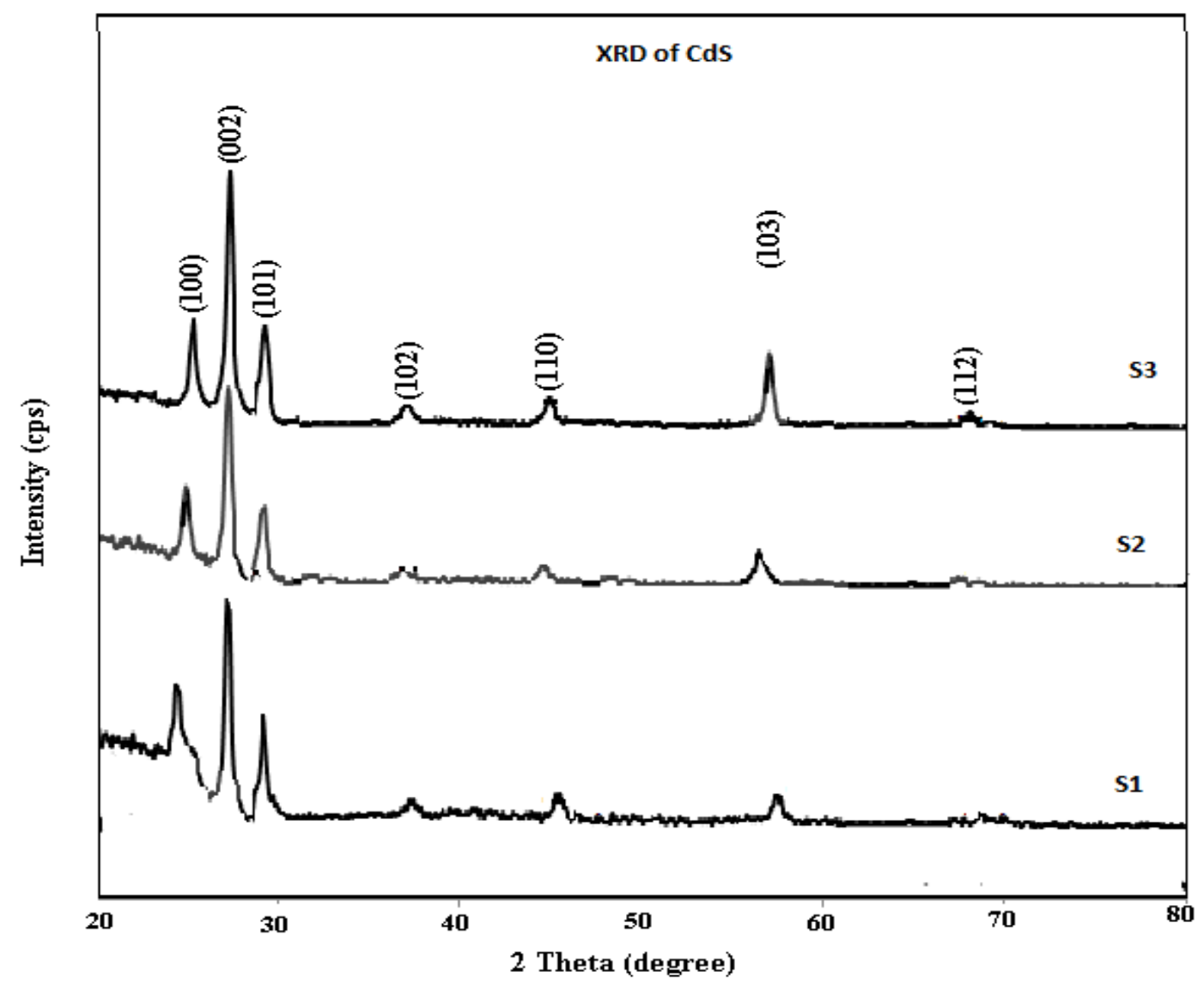

Fig. 1. XRD of the CdS thin sample.

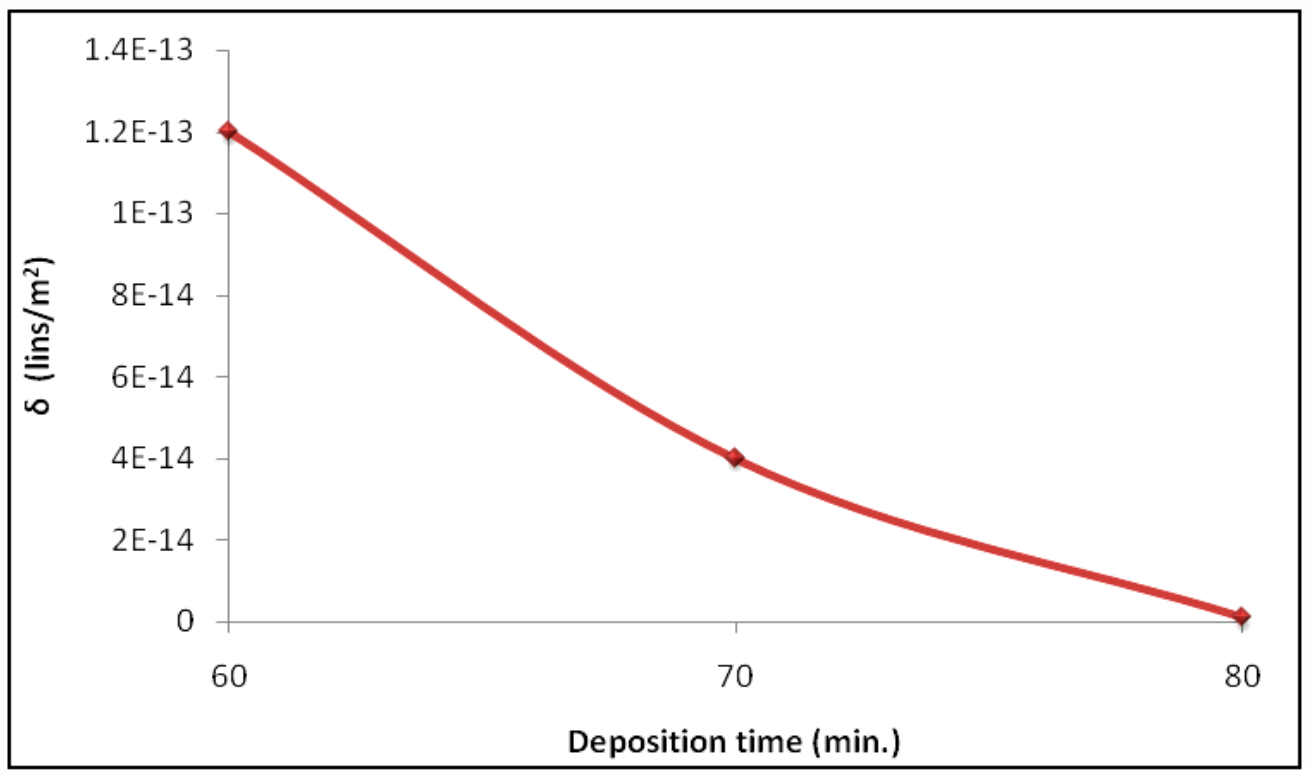

Fig. 2. Dislocation density as a function of deposition time. 
The dislocation density decreases with deposition time as shown in Figure 2. The decreasing is sharp with short deposition time while in longer time the inverse change takes place. Such behavior could be due to the change in grain size accompanied short and longer time deposition.

The number of crystallites per unit area $(\mathrm{N})$ of the films were determined with the use of the following formulae:

Number of crystallites per unit area $(\mathrm{N})=\mathrm{T} / D^{3}$

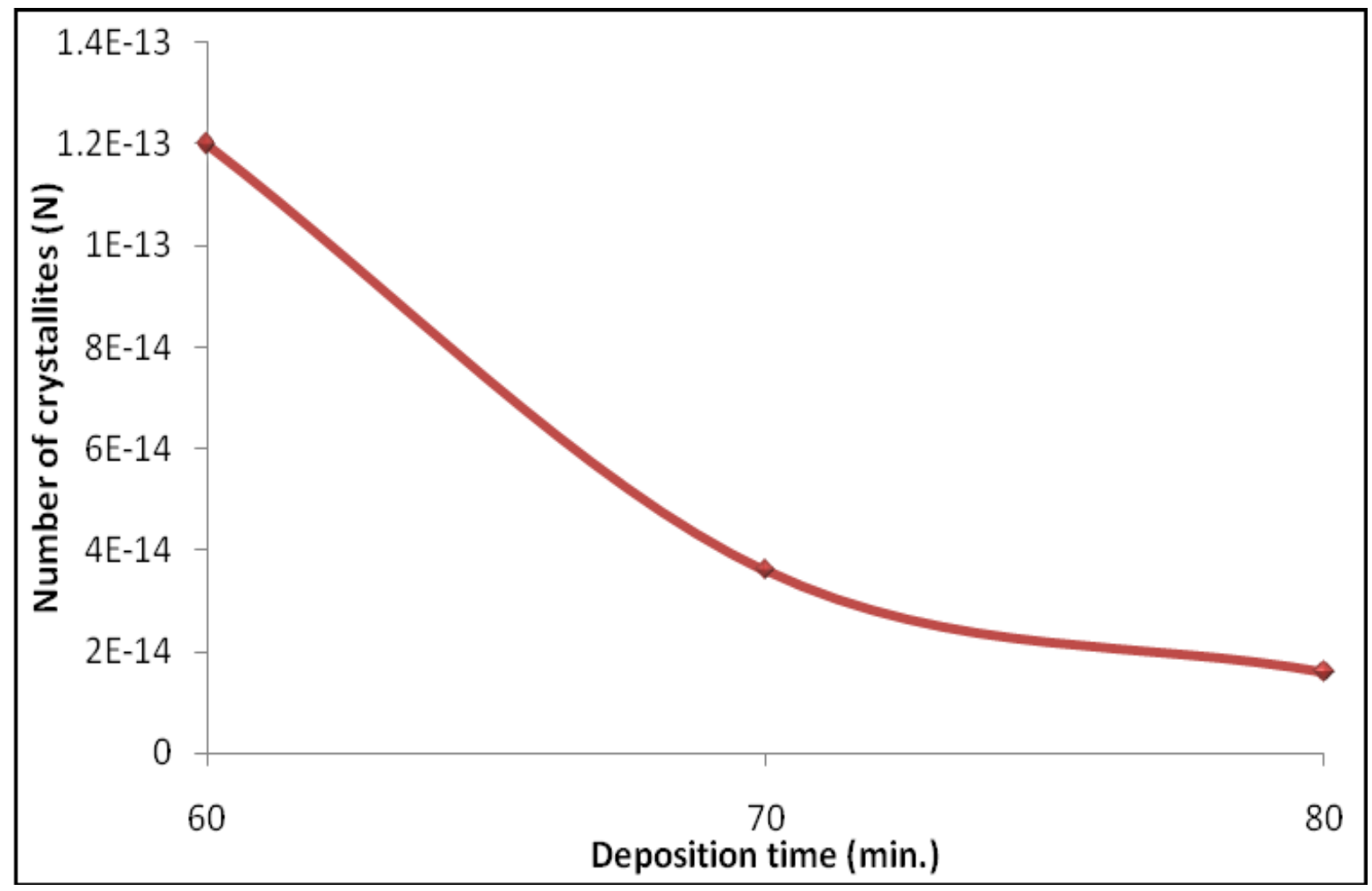

Fig. 3. Number of crystallites as a function of deposition time.

The decreasing in number of crystallites with deposition time is shown in Figure 3 . The decreasing accompanied is that the nucleation with various orientations can be formed at the initial stage of the deposition and each nucleus competes to grow but only nuclei having the fastest growth rate survive.

\section{2. Elemental Analysis by EDAX}

The quantitative elemental analyses of $\mathrm{CdS}$ films were determined using energy dispersive analysis (EDAX) technique at room temperature. Table 2 shows the elemental composition of the films determined by EDS. 


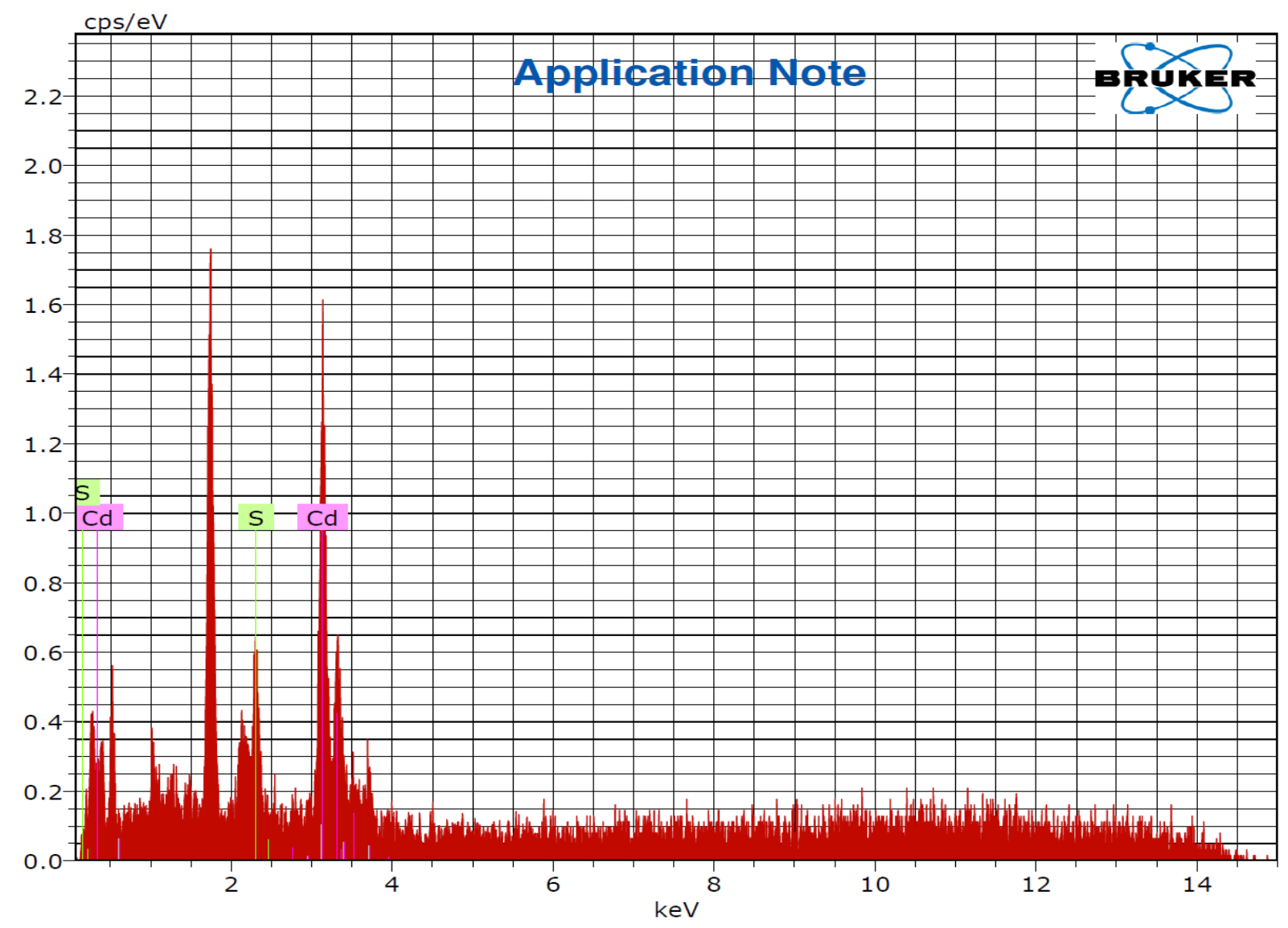

Fig. 4. Elemental analysis of most sensitive nanostructured CdS thin film sample (S2).

Table 2. Elemental composition of nanocrystalline CdS thin films.

\begin{tabular}{|c|c|c|c|c|c|c|}
\hline \multirow{2}{*}{ Element } & \multicolumn{5}{|c|}{ Observed } \\
\cline { 2 - 7 } & \multicolumn{2}{|c|}{ S1 } & \multicolumn{2}{c|}{ S2 } & \multicolumn{2}{c|}{ S3 } \\
\cline { 2 - 7 } & wt \% & at $\%$ & wt \% & at $\%$ & wt $\%$ & at \% \\
\hline S & 35.50 & 42.80 & 31.21 & 64.71 & 29.12 & 45.40 \\
\hline Cd & 64.50 & 57.20 & 68.79 & 35.29 & 70.88 & 54.60 \\
\hline Total & 100.00 & 100.00 & 100.00 & 100.00 & 100.00 & 100.00 \\
\hline
\end{tabular}

Theoretically expected stoichiometric composition of CdS in terms of at \% is: $\mathrm{Cd}=50$ $\%, \mathrm{~S}=50 \%$. It is clear from Table 2 that the films were nonstoichiometric in nature. 
3. 3. Microstructural studies using FE-SEM

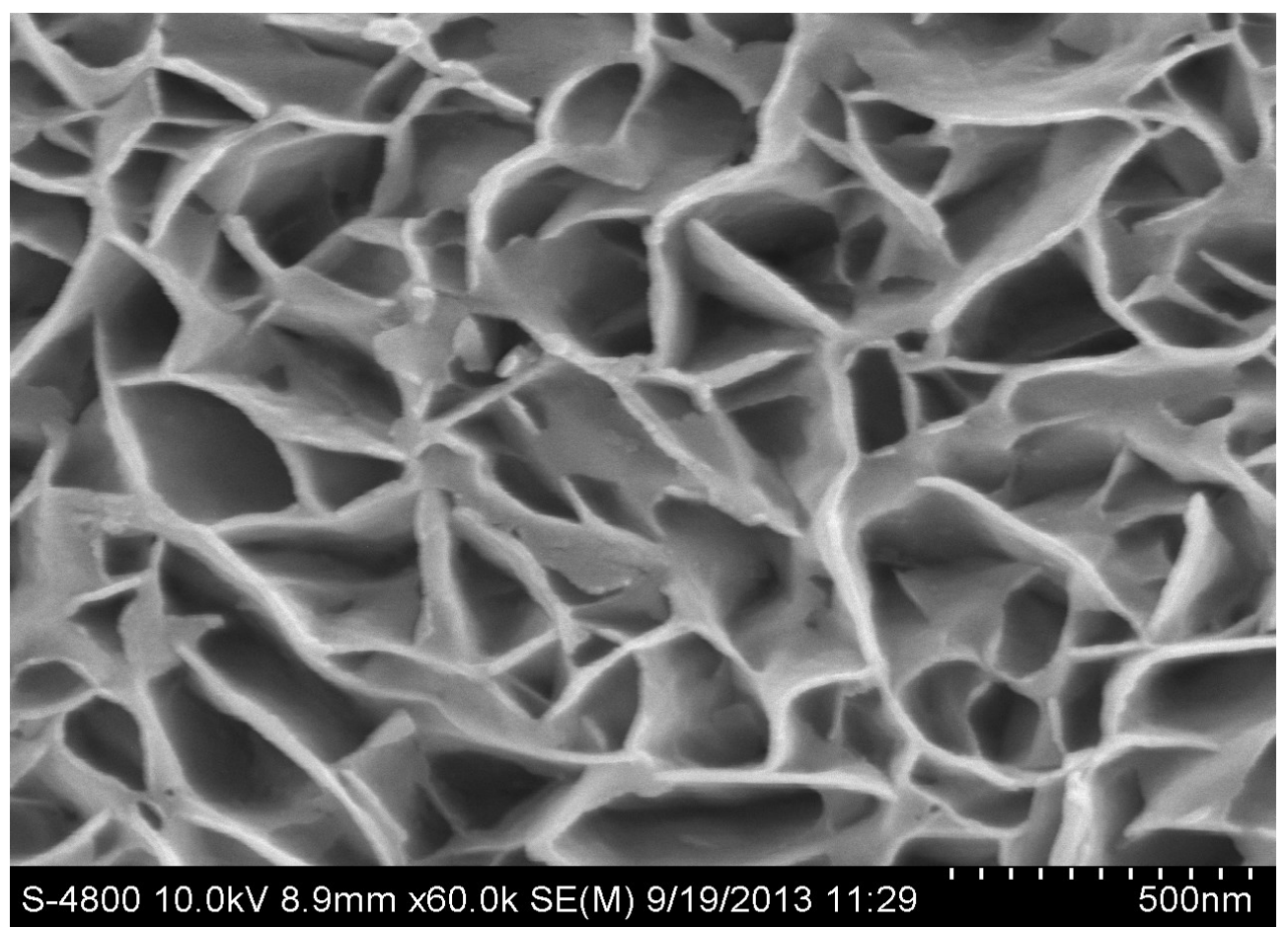

(a)

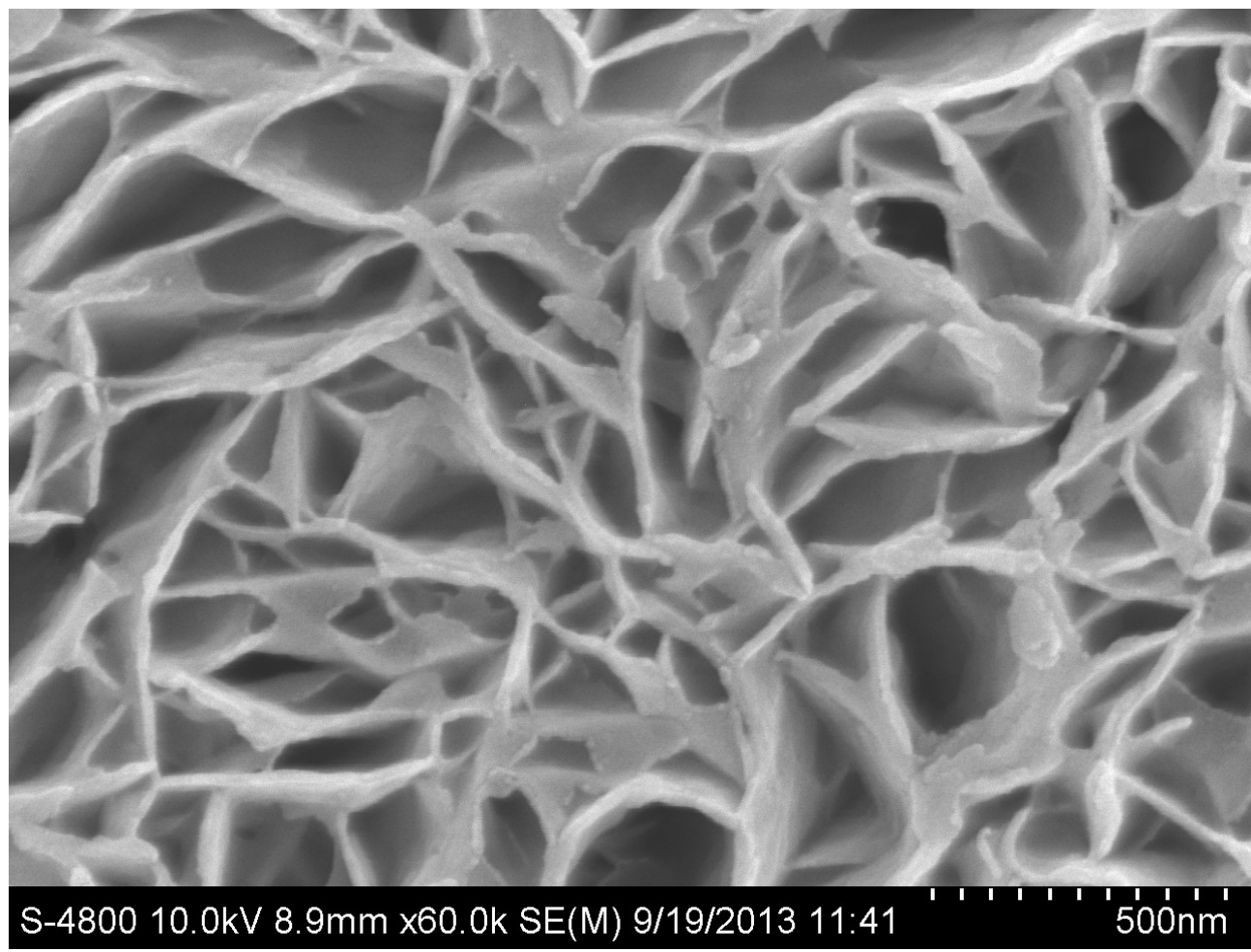

(b) 


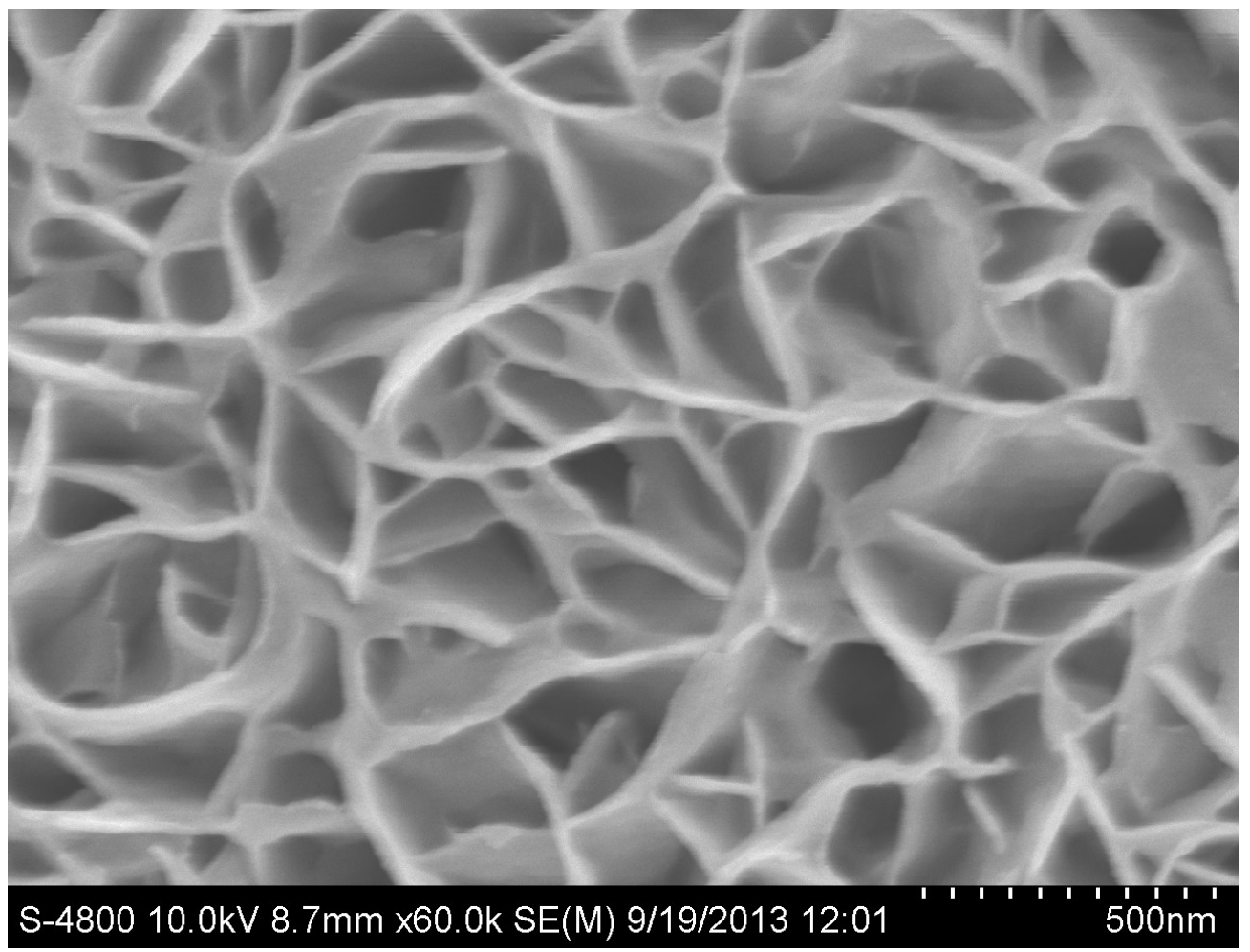

(c)

Fig. 5 (a), (b), (c): FE-SEM images of the CdS samples.

Figure 5 consists of FE-SEM images representing surface morphology of the as synthesized cadmium sulphide films with different thickness of the thin films. The film is homogeneous, well adherent and covers glass substrate without cracks and pin hole. It was observed that the film was uniform yellowish and well substrate covered with homogeneous hexagonal morphology structure.

Table 3. Measurement of film thickness with crystalline and grain size.

\begin{tabular}{|c|c|c|c|c|}
\hline Sample & Deposition & Thickness & Crystallite size & calculated from \\
No. & time & (nm) & XRD & from FE-SEM \\
& (T) min. & & (nm) \\
\hline S1 & 60 & 788 & 31.11 & 29.13 \\
\hline S2 & 70 & 867 & 37.25 & 31.45 \\
\hline S3 & 80 & 917 & 41.23 & 33.78 \\
\hline
\end{tabular}


The thickness of the film was varied from 788 to $917 \mathrm{~nm}$. It was found that the thickness of the film increases deposition time. It is also clear from Table 3, that crystallite size, grain size goes on increasing with increase in film thickness [8].

\section{5. Electrical studies}

\section{5. 1. Measurement of electrical conductivity and activation energy}

Electrical conductivity of the CdS thin films was measured by using DC two-probe method in the temperature range of 313-423 K.

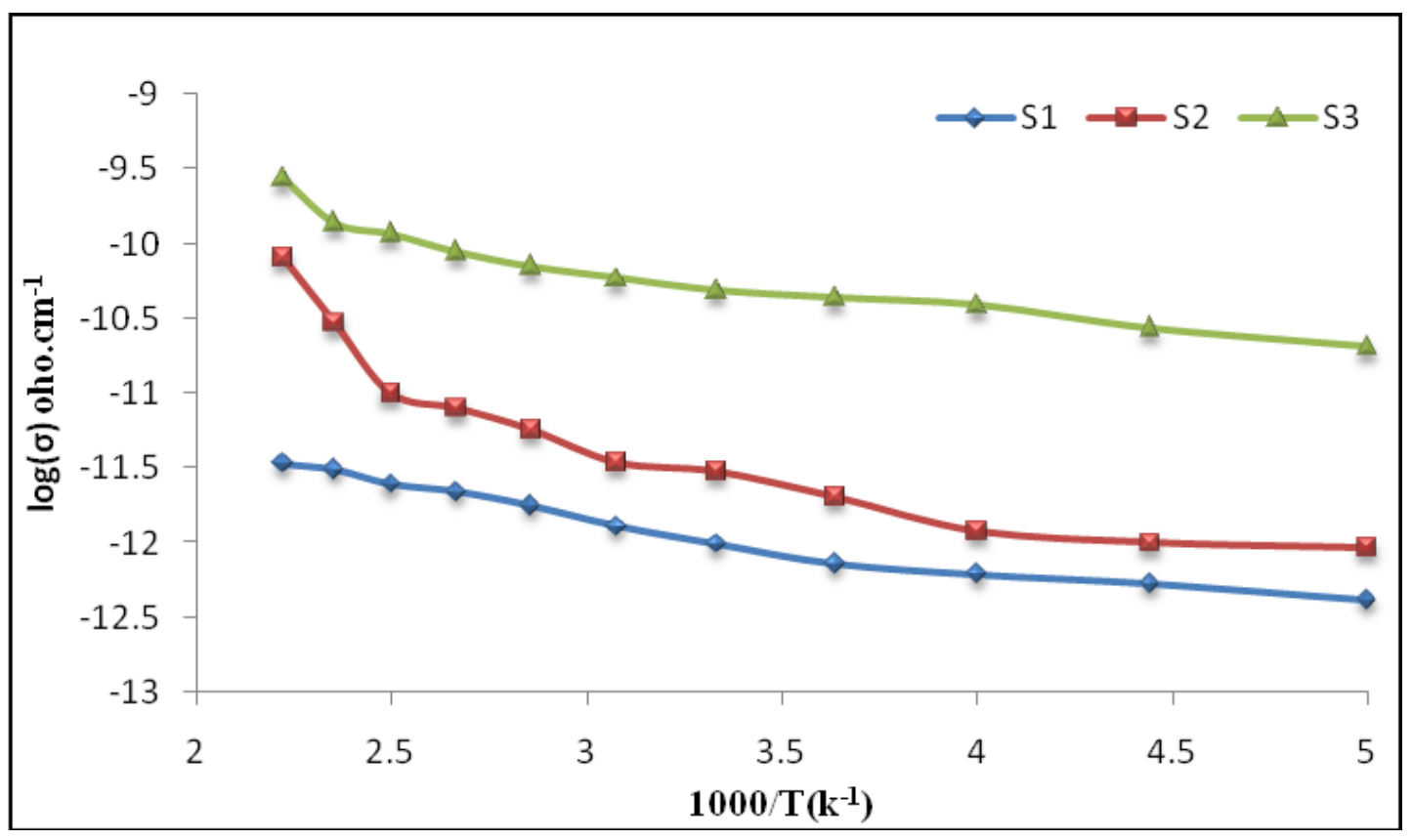

Fig. 6. Variation of electrical conductivity $(\sigma)$ with temperature.

Conductivity was given by relation

where;

$$
\sigma=\sigma_{\mathrm{o}} \exp (-\Delta \mathrm{E} / \mathrm{kT})
$$

$\sigma=$ conductivity

$\sigma_{\mathrm{o}}=$ conductivity constant

$\mathrm{k}=$ Boltzmann constant

$\mathrm{T}=$ Temperature

The conductivity of each sample is observed to be increasing with an increase in temperature.

The increase in conductivity with increase in temperature could be attributed to negative temperature coefficient (NTC) of resistance and semiconducting nature of nanocrystalline CdS. Variation of conductivity with operating temperature (Fig. 6) clearly indicates that the pure nanostructured $\mathrm{CdS}$ films are semiconducting in nature. 
The conductivity studies on films show that all the films exhibit two activation energies at different temperature regions. These activation energies vary with film thickness and are listed in Table 4.

The results indicate the presence of two donor levels - one deep and one shallow near the bottom of the conduction band.

Both of these levels depend on the film thickness. It can be seen that, in high temperature region $(423 \mathrm{~K}$ ) activation energy values increased in all samples.

This is due to the oxygen atoms which are captured at the surface and which form weak links with the free cadmium atoms in the film, are responsible for the electrical conductivity through the surface states.

In the low temperature region $(323 \mathrm{~K})$, the activation energy for $\mathrm{S} 1$ is $0.26 \mathrm{eV}$ is lower than activation energy of samples S2 and S3. This is due to the low concentration of free atoms, in the low temperature range $(323 \mathrm{~K})$.

Table 4. Measurement of film thickness and activation energy.

\begin{tabular}{|c|c|c|c|}
\hline \multirow{2}{*}{ Sample } & \multirow{2}{*}{ Thickness } & \multicolumn{2}{|c|}{ Activation energy } \\
No. & $\mathbf{( n m )}$ & $323 \mathrm{~K}$ & $423 \mathrm{~K}$ \\
& & (Low Temperature) & (High Temperature) \\
\cline { 3 - 4 } & & $0.26 \mathrm{eV}$ & $0.36 \mathrm{eV}$ \\
\hline S1 & 788 & $0.23 \mathrm{eV}$ & $0.27 \mathrm{eV}$ \\
\hline S2 & 867 & $0.19 \mathrm{eV}$ & $0.23 \mathrm{eV}$ \\
\hline S3 & 917 & & \\
\hline
\end{tabular}

It is reported $[9,10]$ as the thickness of the film increases activation energy goes on decreasing. It is clear from Table 4 that, as film thickness of the sample goes on increasing; the activation energy goes on decreasing.

The decrease in activation energy with increasing film thickness may be due to the change in structural parameters, improvement in crystallite and grain size [11].

\section{5. 2. Thermoelectric power}

Thermoelectric power (TEP) versus temperature curves are shown in Figure 7 for different thickness of CdS. It is clear from figure that TEP goes on increasing with increase in temperature for all the samples. TEP is negative for all samples during the temperature range of 320-440 K, indicating n type semiconductivity.

Figure 7 shows variation of TEP temperature (k). It is clear from Figure 7 that TEP becomes more negative with increase in thickness if the films. 


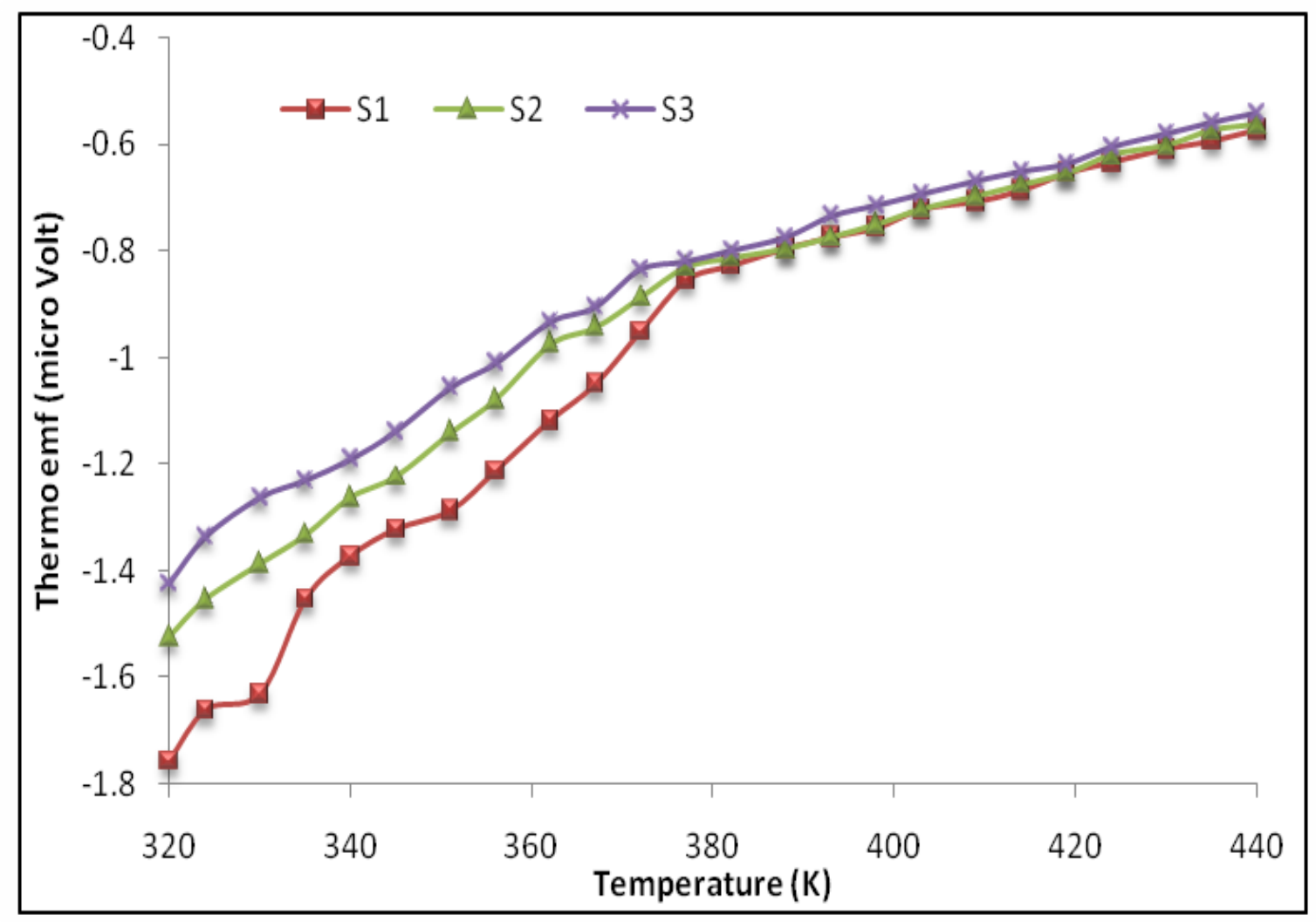

Fig. 7. TEP verses temperature (k) profile.

\section{5. 3. Photoconductivity studies}

When excess electrons and holes are created in a semiconductor, there is corresponding increase in the conductivity of the sample. If excess carriers arise from optical excitation, the result in increases in the conductivity. This is an important effect, with useful application in the analysis of semiconductor materials and in the operation of several types of devices.

There are number of applications for devices, which change their resistance when exposed to light. Such light detectors can be used in the home to control automatic night light which turned on at dusk and turn off at down and also used to measure illumination levels, as in exposure meters for cameras.

Detectors are used in optical signaling systems in which information is transmitted by a light beam and is received at a photoconducting cell.

Consideration in choosing a photoconductor for a given application include the sensitive wavelength range, time response and optical sensitivity of the material.In general, semiconductor are most sensitive to photons with energies equal to the band gap or slightly more energetic than band gap. Less energetic photons are not absorbed, and photons with hv $>\mathrm{Eg}$ are absorbed at a surface and contribute to bulk conductivity.

For general $\mathrm{CdS}(\mathrm{Eg}=2.42 \mathrm{eV})$ is commonly used as a photoconductor in the visible range. Experimental set up for photoconductivity measurement as shown in Figure 8. 


\section{Light Radiation}
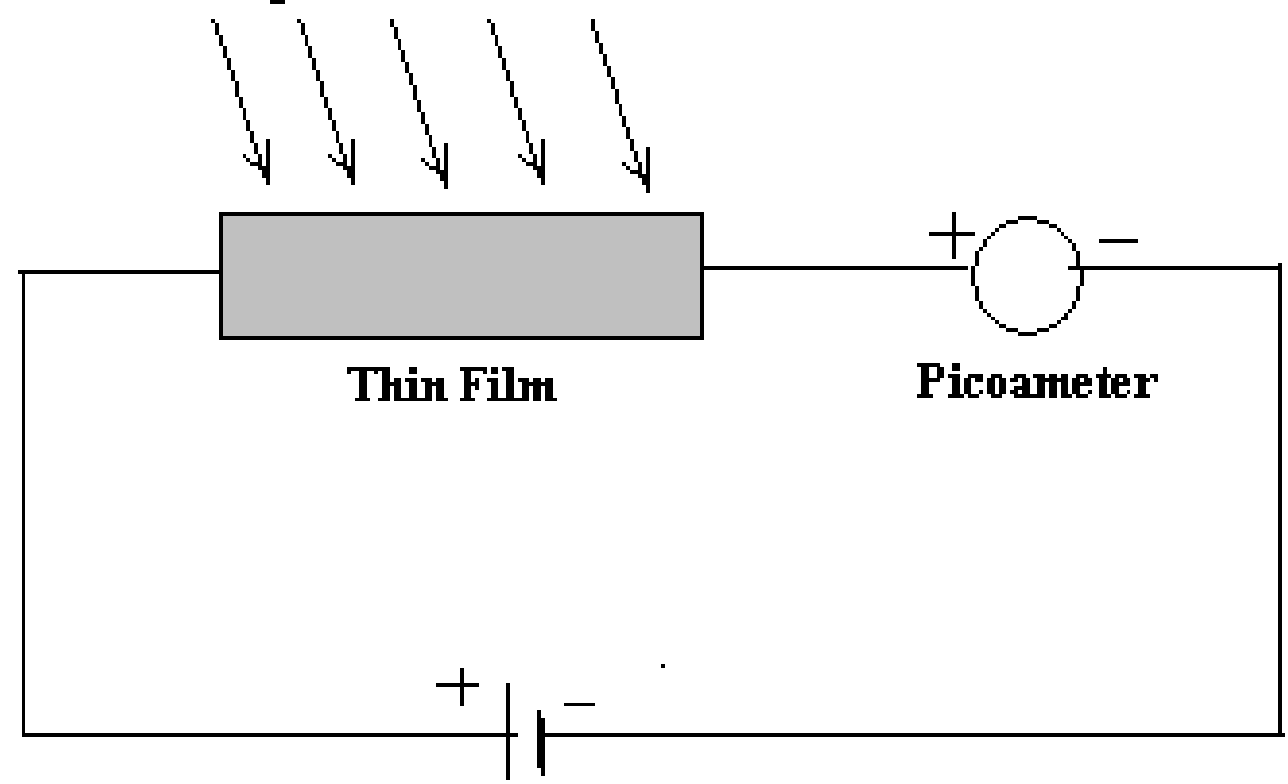

High Voltage Power Supply

Fig. 8. Circuit diagram for Photoconductivity Measurement.

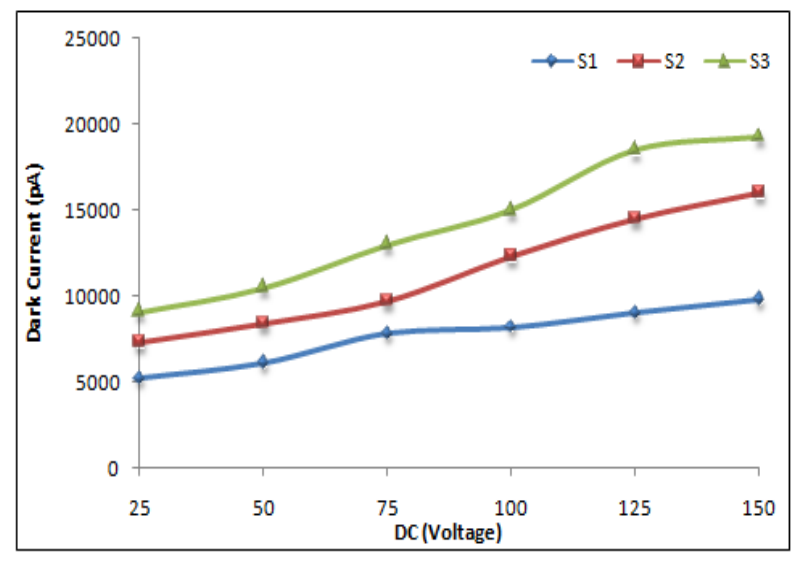

(a)

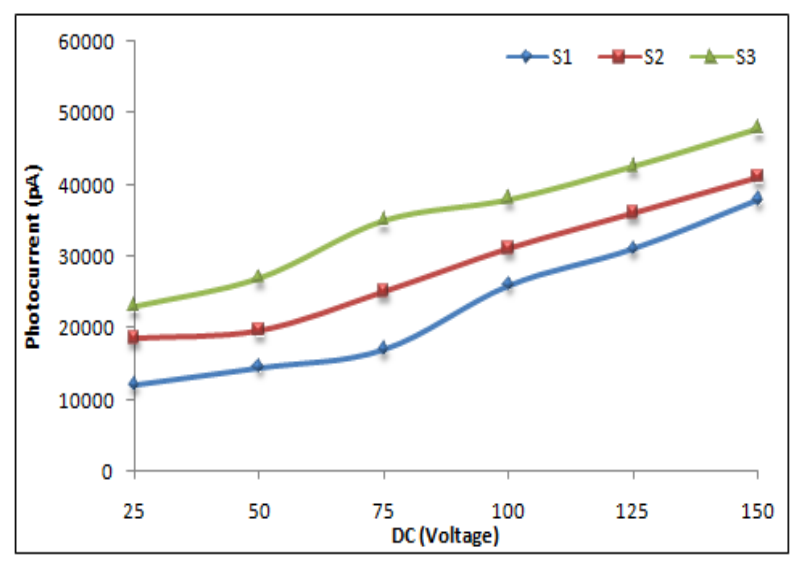

(b)

Fig. 9. (a) and (b) Photocurrent Vs applied voltage characteristics for Dark and Illuminated light intensity.

Fig. 9 (a) and (b) shows the variation of photocurrent of CdS thin films with applied voltage for dark and illuminated light intensity. The plot shows that there is a linear dependence of photocurrent with applied voltages. It was observed that photoconductivity increases when light incident on film, the CdS films have the highest conductivity in presence of light incident. 


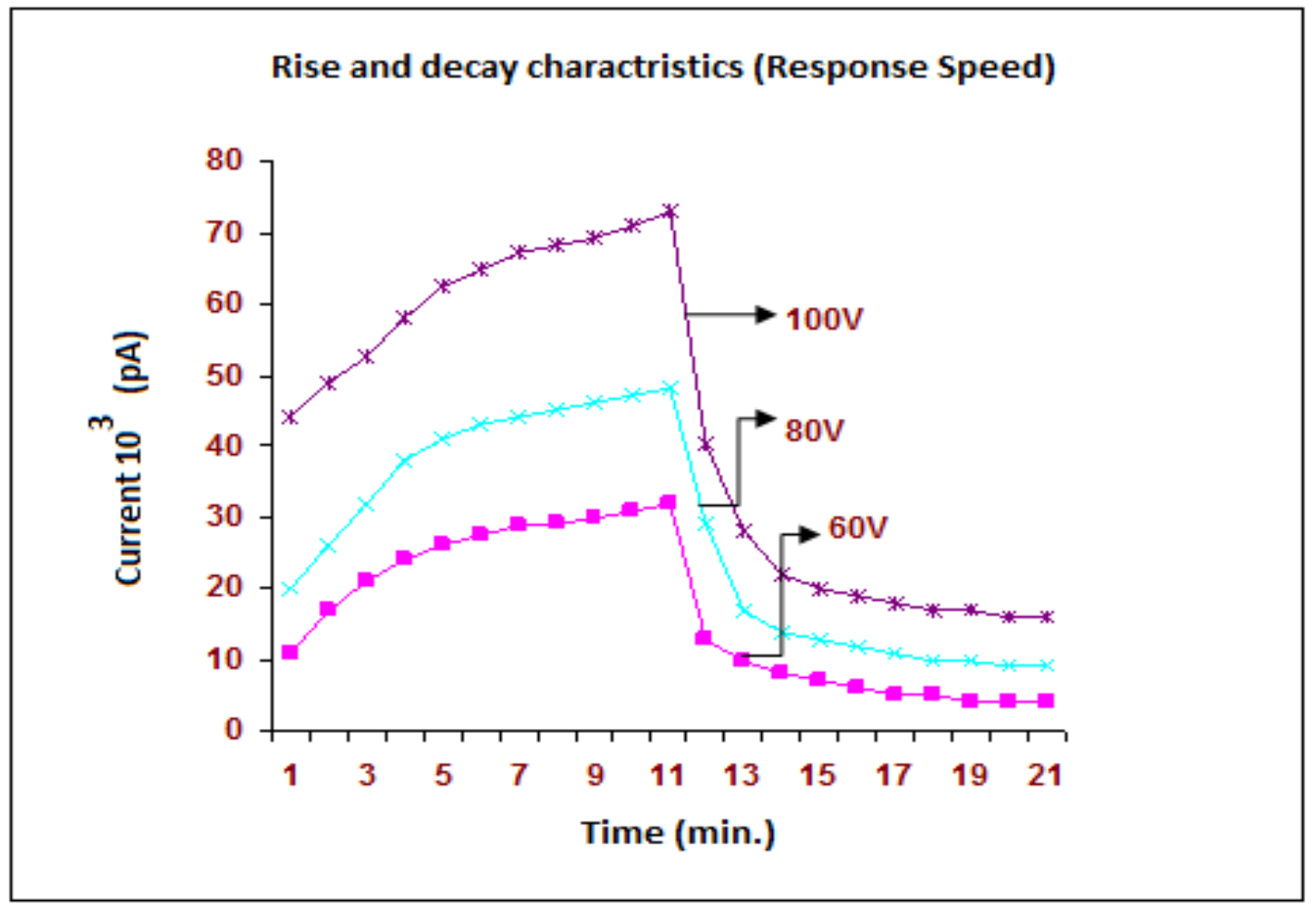

Fig. 10. Response time of thin film $($ Sample $=$ S2) at different voltage.

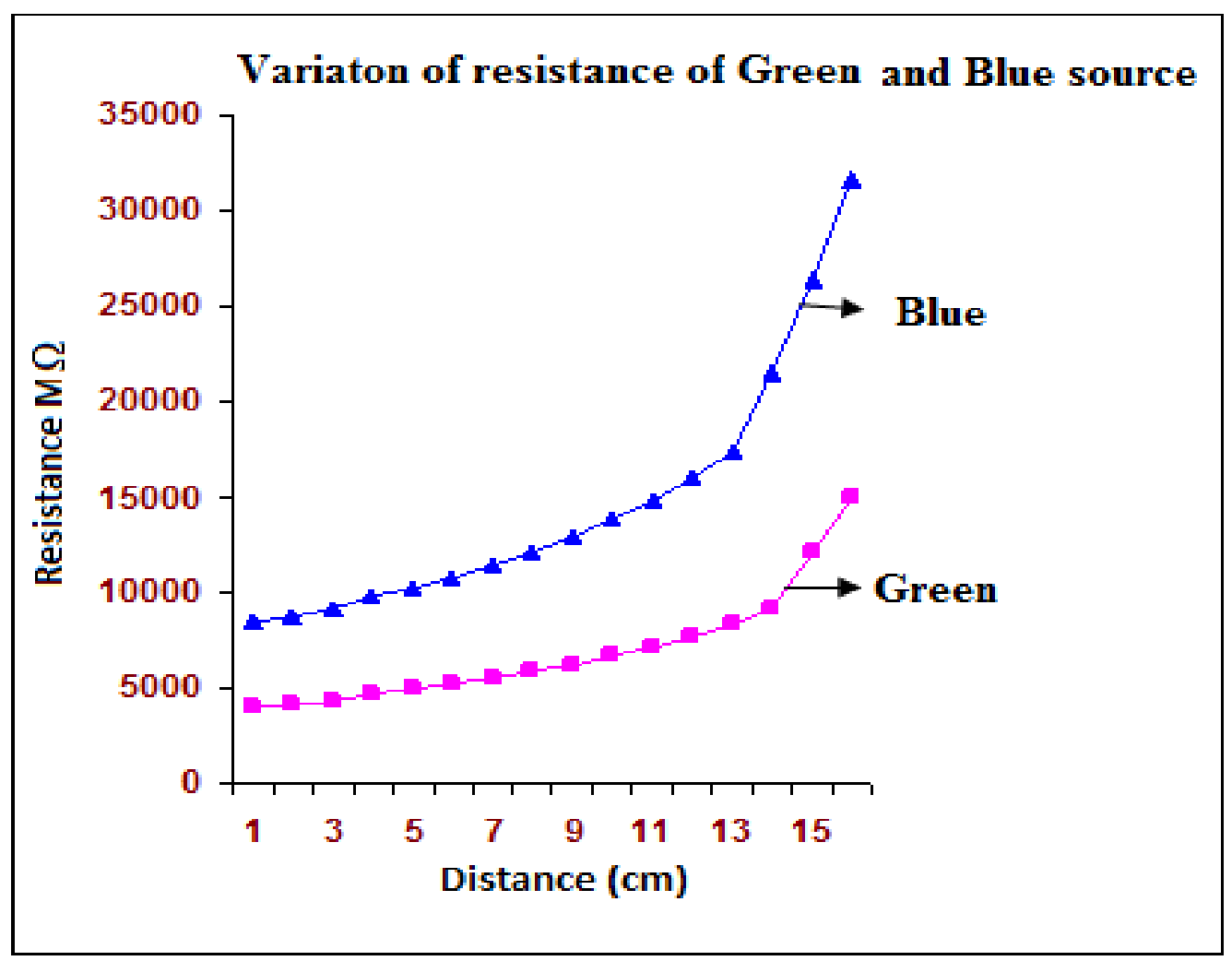

Fig. 11. Variation of resistance with distance for different sources of light. 
The response and recovery of thin film (Sample $=$ S2) on exposure of at different operating voltage (60-100 V) are represented in Figure 10. If we plot a graph of photocurrent against time (in $\mathrm{min}$ ), it show rise \& decay characteristics of thin film. Initially it increases \& attains peak value, when light is incident on film i.e. when it is ON. But photocurrent decreases even though time increases it means that when it is OFF i.e. the photoconductive film work as light dependent switching action. The response is quick (11 min.) and recovery is fast (10 min.). Fig. 11 shows increase in resistance as distance increases. It is also observed that resistance is high for blue source than green source. Therefore we can conclude that resistance for green light is low as compare to blue light [12-22].

\section{CONCLUSIONS}

1) Nanocrystalline cadmium sulphide films were deposited on to glass substrate by simple chemical bath deposition technique.

2) The films obtained were uniform and had good adherence to the substrate.

3) The EDAX of the film indicated that the films were nonstoichiometric.

4) It was found that as increase in dipping time of bath, thickness of the films, crystalline size and grain size goes on increased with decreased in dislocation density and number of crystallites

5) The activation energies were decreasing with the increase in thickness of the films.

6) The increase in conductivity with the increase in temperature indicated that the films were semiconducting in nature.

7) Negative values of TEP indicated that the films were $n$ type.

8) The nanocrystalline CdS thin films exhibits rapid response-recovery which is one of the main features of this semiconducting thin sample.

9) Study of photoconductivity indicates that, photocurrent in presence of light is high as compare to dark light it show resistance towards blue light is high as compared to green light.

\section{Acknowledgements}

The authors are thankful to Head, Department of Physics and Principal, Z.B.Patil, Arts, Science and Commerce College, Dhule for providing laboratory facilities for this work. Thanks to Principal, S. S.M.M. College, Pachora for his encouragement.

\section{References}

[1] R. H. Bari, V. Ganesan, S. Potadar, L. A. Patil, Bull. Mater. Sci. 32(1) (2009) 37-42.

[2] J. Han, C. Spanheimer, G. Haindl, G. Fu, V. Krishnakumar, J. Schaffner, C. Fan Zhao, K. Klein, W. Jaegermann, Sol Energy Mater Sol Cells 95 (2011) 816-820.

[3] U. M Jadhav, S. N. Patel, R. S. Patil, Research Journal of Material Sciences 1(1) (2013) 21-29.

[4] K. Lingeswaran, S. S. Prasad Karamcheti, M. Gopikrishnan, G. Ramu, Middle-East Journal of Scientific Research 20(7) (2014) 812-814.

[5] B. S. Rao, B. R. Kumar, V. R. Reddy, T. S. Rao, Chalcogenide Letters 8 (2011) 177-185. 
[6] A. E. Rakhshani, A S Al-Azab, J. Phys.: Condense. Matter 12 (2000) 8745-8755.

[7] G. E. Patil, D. D. Kajale, S. D. Shinde, R H. Bari, D. N. Chavan, V. B. Gaikwad, G. H. Jain, Sensors \& Transducers Journal 9 (2010) 96-108.

[8] V. B. Patil, G. S. Shahane, L. P. Deshmukh, Materials Chemistry and Physics 80 (2003) 625-631.

[9] K. C. Sharma, J. C. Garg, Phys. D: Appl. Phys. 23 (1990) 1411-1420.

[10] Z. S. EL-Mandouh, M. EL-Shabasy, Fizikaa 4 (1995) 17-31.

[11] R. B. Kale, C. D. Lokhande, Semicond. Sci. Technol. 20 (2005) 1-9.

[12] Hanan R. A. Ali, International Letters of Chemistry, Physics and Astronomy 8 (2014) 47-55.

[13] Raghad Y. Mohammed, S. Abduol, Ali M. Mousa, International Letters of Chemistry, Physics and Astronomy 10 (2014) 91-104

[14] Raghad Y. Mohammed, S. Abduol, Ali M. Mousa, International Letters of Chemistry, Physics and Astronomy 11(2) (2014) 146-158.

[15] S. M. Patil, P. H. Pawar, International Letters of Chemistry, Physics and Astronomy 17(1) (2014) 21-36.

[16] T. Borowski, Asian Journal of Chemistry 21(6) (2009) 4932-4936.

[17] Nadir Fadhil Habubi, Sami Salmann Chiad, Saad Farhan Oboudi, Ziad Abdulahad Toma, International Letters of Chemistry, Physics and Astronomy 4 (2013) 1-8.

[18] Saad F. Oboudi, Nadir F. Habubi, Ghuson H. Mohamed, Sami S. Chiad, International Letters of Chemistry, Physics and Astronomy 8(1) (2013) 78-86.

[19] J. A. Najim, J. M. Rozaiq, International Letters of Chemistry, Physics and Astronomy 10(2) (2013) 137-150.

[20] Majid H. Hassouni, Khudheir A. Mishjil, Sami S. Chiad, Nadir F. Habubi, International Letters of Chemistry, Physics and Astronomy 11 (2013) 26-37.

[21] K. K. Patankar, International Letters of Chemistry, Physics and Astronomy 1 (2014) $1-8$.

[22] Raghad Y. Mohammed, S. Abduol, Ali M. Mousa, International Letters of Chemistry, Physics and Astronomy 10 (2014) 91-104. 